\title{
ECKART PANKOKE
}

\section{Sinn und Form freien Engagements}

\author{
Soziales Kapital, politisches Potential und reflexive Kultur \\ im Dritten Sektor
}

Die heute als "Dritter Sektor" diskutierte Selbstorganisation bürgerschaftlichen Gemeinsinns verstand sich schon immer als Antwort auf Krisen und Schwellen sozialen Wandels. Soziologisches Interesse richtet sich dabei auf die Organisationsformen des auf "Gemeinschaften" bezogenen sozialen Kapitals und politischen Potentials freier Vereinigungen und Bewegungen, aber auch auf die Sinnkonstruktionen von Ehre und Engagement. In gesellschaftsgeschichtlicher Perspektive ist dazu bewußt zu machen, wie im Zuge gesellschaftlicher Modernisierung auch die Sinnkonstruktionen und Organisationsformen gemeinsinnigen Engagements sich wandelten: von der Traditionalität zur Rationalität, von der Repräsentation zur Reflexivität.

Historisch weisen die Programme und Probleme bürgerschaftlicher Autonomie weit zurück vor die bürgerliche Moderne: Im Ordnungsdenken und Gemeinschaftsleben des alten Europa fanden viele der heute noch beschworenen Leitbegriffe aktiven Gemeinsinns und freien Engagements ihre traditionelle Prägung: „Ehre“ und „Treue“, „Innung“ und „Stiftung", ,Verein" und „Verband“, „Gemeinschaft" und „Genossenschaft", „Corporation" und „Communio", „Gemeinnutz" und „Gemeingeist".

Erst die Modernisierungsschwellen des Europäischen Revolutionszeitalters machten die Ordnungen des Sozialen zum Problem der Organisation von Gesellschaft. Auch die Formen politischer und sozialer Verfaßtheit wurden jetzt bewußt als Errungenschaften institutioneller Konstruktion. Erst jetzt war moderne Wirklichkeit „selbst“ zu erarbeiten und $\mathrm{zu}$ verantworten in ihrer Geschichtlichkeit, ihrer Gestaltbarkeit und ihrer Künstlichkeit. Entsprechende Programmformeln, die sich im 19. Jahrhundert durchsetzten bzw. neu an Bedeutung gewannen, waren "Selbständigkeit", „Selbstverwaltung", "Selbsthilfe".

Eine heute sich weitende Pluralität und Komplexität moderner Gesellschaften fordert auch in ihrer Steuerung und Selbststeuerung einen Perspektiven-, ja Paradigmenwechsel von kontrollierter Rationalität zu (selbst-) bewußterer Reflexivität. Das hat auch Folgen für den Stellenwert des „Dritten Sektors“, dem Kritiker unterstellen, daß er in ,funktionalem Dilettantismus ${ }^{61}$ hinter den Anforderungen der Rationalisierung zurückblieb, von

I Seibel 1992. 
dem andere aber (gerade deshalb) erwarten, daß Gemeinsinn in der Reflexivität sozialer Selbststeuerung auf den Wegen in eine ,andere Moderne“ zur treibenden Kraft wird.

\section{Ständische Freiheiten und bürgerliche Autonomie}

Die Programmgeschichte gesellschaftlicher Selbstorganisation spiegelt die Krisen und Schwellen im Prozeß der Modernisierung. Dies ist aufzuzeigen an der institutionellen Genese ,gemeinsinniger‘ Ämter und Ehren in Stiftungen, Genossenschaften, Selbstverwaltungen - aber auch an der Entwicklung freien Engagements in freien Vereinigungen und sozialen Bewegungen.

In seiner soziologischen Rekonstruktion moderner Vergesellschaftung faßte Max Weber die korporative Autorität und Autonomie der „Rechtspersönlichkeit" als frühe Grundform bürgerlicher Selbstorganisation. Dabei würdigte Weber das Institut der ,Stiftung ${ }^{*}$ - sowohl gegenüber den politischen Interessen staatlicher Herrschaft wie gegenüber den wirtschaftlichen Eigen-Interessen ihrer Akteure - als Grundtypus institutioneller Autonomie: In der frühen Selbstbehauptung „ständischer Freiheiten“ gegenüber weltlicher Herrschaft war für Weber die moderne System-Trennung von "Staat" und "Gesellschaft" vorbereitet - als institutioneller Rahmen, der die Ausdifferenzierung gesellschaftlicher Selbstorganisation erst möglich machte. Demnach begründete sich der Status ,moderner“ Autonomie bei den ersten Stiftungen traditionell über die Widmung an einen „heiligen“ Zweck - oft verkörpert im Stiftungs-Patron, dessen „Heiligkeit" gegenüber weltlicher Gewalt fundamentale „Freiheit" bedeutete und beanspruchte. In der frühen Neuzeit trat dann an die Stelle der Autorität des ,Heiligen“ die säkulare Autonomie bürgerlicher ,Ehren“:

„Im Okzident wurde zunächst der Heilige als Eigentümer des Stiftungsgutes behandelt und begann sich ein weltlicher Stiftungsbegriff des Mittelalters zu entwickeln, nachdem das kanonische Recht ihn für weltliche Zwecke vorbereitet hatte. “2

Dabei wurde der Stiftungszweck transformiert aus dem Eigensinn des Stifters in den Gemeinsinn der gemeinnützigen „Stiftung“. „Stiftung“ bedeutete zudem die generationenübergreifende Beständigkeit der durch gemeinsamen Grund und Sinn gebundenen Gemeinschaft. Diese Bedeutungsspannung von "Grundlegen", „in Ordnung halten", aber auch von ,in Bewegung bringen" bestimmt auch das moderne Verständnis von „Stiftung" in der Spannung von Bewahrung und Erneuerung. ${ }^{3}$

Stiftungen wie auch andere Initiativen eines freien Gemeinsinns stehen heute vor dem Problem, daß die treuhandliche und ehrenamtliche Wahrung von Erbe und Vermächtnis in einer sich wandelnden Gesellschaft gefordert ist, immer wieder auf neue Probleme und neue Potentiale der gesellschaftlichen Umwelt (inter)aktiv Bezug zu nehmen. Die repräsentative Kultur des traditionellen Ehrenamtes verbindet sich dann - gerade im Handlungsfeld der , operativen Stiftung * - mit der Reflexivität strategischen Lernens.

2 Weber 1964, S. 548

3 Vgl. Pankoke 1998. 
Eine andere Traditionslinie europäischer Gemeinschaftsformen weist zurück auf das Institut der „Innung“ als Konstruktion verbandlicher Einigkeit durch „Einigung“ der Mitglieder. So wird bis heute noch in traditionellen Vereinskulturen an den für viele längst altertümlichen Parolen der „Innigkeit“ und „Eintracht" festgehalten. Diese Beschwörung des „Innungs-Geistes" - so Ernst Moritz Arndts patriotischer Aufruf zu gemeinsinnigen Vereinigungen („Deutscher Gesellschaften“) im „Entwurf einer teutschen Gesellschaft" (Frankfurt/M. 1814) - gewann für die politische Kultur des Vereinswesens programmatische Tradition.

Doch im Zuge der Modernisierung zeigte sich, wie die Ordnungen und Bindungen korporativer ,Eintracht" locker wurden, um zugleich die Schwelle frei zu machen für eine radikale Modernität ,sozialer Bewegung“ und eine sich steigernde Reflexivität der Assoziationen ,freien Engagements“.

\section{Ehre und Engagement: zwischen Enthusiasmus und Dilettantismus}

Der Begriff der Ehre - im indo-germanischen Bedeutungsfeld von ,ais“" (,Erz, glänzendes Metall') - spiegelt den Glanz und zugleich die Härte dessen, was auf Dauer setzt. Dies verband sich mit der Bedeutung von ,Ehre' als Ethos des Ortes: Es galt die Erwartungen eines sozialen Raumes in dessen Mitte (,milieu') so auszufüllen, daß die geteilten Werte eines gemeinsamen geschichtlichen Grundes ,verkörpert" wurden.

Um 1800 markierten dies die damals modernen Programmformeln, die bald zu Problemformeln wurden: ,Enthusiasmus" und ,Dilettantismus', wobei ,Enthusiasmus' ursprünglich bedeutete, daß in einem Menschen ,göttlicher Geist" wirkt, dessen ,Begeisterung' begeisternd ausstrahlt. Im Zuge der Modernisierung aber ging aufklärende ,Entzauberung auf kritische Distanz zu jeglichem ,Enthusiasmus", der nun als weltfremde ,Schwärmerei" verworfen wurde. Auch der „Dilettantismus" markierte zunehmend das abwertende Defizit fehlender Kompetenz, während die ursprüngliche Bedeutung, wie es in der italienischen Übersetzung der Ehrenamtlichen als "dilettanti" noch mitklingt, daß hier ,aus Freude“ (,diletto") gehandelt wird und diese Freude letztlich in Freiheit gründet, vergessen wurde. ${ }^{4}$

Der klassische Zusammenhang von ,Freiheit' des Amtes und ,Freude' des Handelns, also - modern gesprochen - von ,Engagement" und ,Autonomie ${ }^{6}$ ist zu übersetzen in aktuelle Personal- und Organisationsprobleme des ,Dritten Sektors`. Doch wäre dem kritischen Vorwurf eines, funktionalen Dilettantismus ${ }^{6} \mathrm{zu}$ erwidern, daß ein gegenüber funktionalen Zwängen und Interessen eigenständiges und selbstbewußtes Engagement von Ehrenamtlichen eine ,extra-funktionale Freiheit‘ behaupten könnte.

4 Siehe dazu Pankoke 1994. 


\section{3. „Labyrinth der Bewegung“: Emanzipation und Assoziation}

Im „Labyrinth der Bewegung" der industriellen Revolution waren „Gemeinsinn“ und „Gemeinwohl“ neu zu beziehen auf die sich krisenhaft verschärfenden Probleme sozialer Verelendung und Entfremdung: Soziale Not wurde nun prinzipieller gefaßt als Systemfrage der gesellschaftlichen Entwicklung und Steuerung. Jetzt war das Elend von Pauperismus und Proletarisierung nicht mehr hinzunehmen als göttliches Geschick oder individuelles Schicksal, sondern zu erklären als Folge der Organisations- und Konstruktionsprinzipien des herrschenden Systems.

Das neue Losungswort der Suche nach Lösungen der Emanzipations-Krise hieß ,Association": Dies sollte den institutionellen Rahmen markieren zur „Einbürgerung des Proletairs". 5 Vor dem Hintergrund der mit dem Industrialisierungsprozeß verbundenen Problemverlagerung vom Pöbel zum Proletariat (Conze) sprach Karl Marx damals in einem radikaleren Sinne von ,künstlicher Armut". Das moderne Bewußtsein der Künstlichkeit gesellschaftlicher Verhältnisse setzte auf die Machbarkeit von revolutionären Lösungen des Problems proletarischer Entfremdung. Dabei erkannte Marx in der Zerschlagung älterer Gemeinschaftsbindungen die Voraussetzung des sich von allen Rücksichten emanzipierenden ökonomischen Egoismus:

„Die politische Revolution, [...] zerschlug notwendig alle Stände, Korporationen, Innungen, Privilegien [...]. [...] Die Abschüttlung des politischen Jochs war zugleich die Abschüttlung der Bande, welche den egoistischen Geist der bürgerlichen Gesellschaft gefesselt hielten. [...] Die feudale Gesellschaft war aufgelöst in ihren Grund, in den [...] egoistischen Menschen. ${ }^{.6}$

Die sozialrevolutionäre Programmatik der sozialen Bewegung setzte mit ihrer Radikalisierung des Prinzips der „Assoziation" nicht nur die traditionellen „Korporationen“ außer Kraft, sondern beanspruchte auch die Antithese zur industriekapitalistischen „Organisation der Arbeit". „Assoziation" zielte auf neue Sozialformen, welche die modernen Prinzipien der Emanzipation und der Solidarität verbinden sollten. Schon in der frühen Arbeiterbewegung war der in den „Ideen von 1789“ proklamierte Grundwert der „Brüderlichkeit" dynamisiert zum Bewegungsbegriff einer solidarischen „Organisation der Arbeit“. Marx und Engels radikalisierten das dem Programm der „Arbeiterverbrüderung" vorangestellte Motto ,Alle Menschen sind Brüder" im Kommunistischen Manifest durch die klassenkämpferische Parole „Proletarier aller Länder vereinigt Euch!". Am utopischen Horizont erschien nun die neue Gesellschaft als ,große Assoziation“.

Die wissenschaftliche Distanz des geschichts- und gesellschaftstheoretischen Beobachters im „Labyrinth der Bewegung“ beanspruchte Lorenz von Stein: ${ }^{7}$ Für ihn wurde „Bewegung“ zum Schlüssel zeitgeschichtlicher Erfahrung. Dabei gründet Steins Bewertung der "sozialen Bewegungen " in der Beobachtung, daß deren Dynamik eher in der kritischen Negation des herrschenden Systems liegt. Als Wissenschaftler suchte Stein seinen Standpunkt von Gesellschaftslehre und Politikberatung ,über" den Bewegungen. Als eine „über" den Parteiungen stehende Praxis fordert er die ,Republik des gegenseiti-

\footnotetext{
Vgl. Baader 1835 .

Marx/Engels 1983, S $368 \mathrm{f}$.

Stein 1850/1921, Bd. 1, S. 65.
} 
gen Interesses“ im Zusammenspiel von gesellschaftlichem „Vereinswesen“ und „Verwaltung der sozialen Reform".

$\mathrm{Daß}$ im „Labyrinth der Bewegung" noch andere Kräfte sozialen Engagements als „Bewegung" aktiv wurden, zeigt der Blick auf die Anfänge der Frauenbewegung im Schnittfeld von Demokratiebewegung und Arbeiterbewegung: Publizistisches Forum war die von Louise Otto herausgegebene „Frauen-Zeitung“(1849/50), in der neben den Fragen der Frauenemanzipation auch die soziale Lage der Arbeiterinnen als Bezugsproblem „sozialer Bewegung“ publik wurde. Vor allem die Artikelfolge „Die Demokratinnen" gab Zeugnis einer zeitbewußten Selbstbeobachtung und Selbstverständigung ,sozialer Bewegung،.

Die innere Bewegtheit durch sozial wie politisch engagierten Gemeinsinn faßte Louise Otto im Typus der „Enthusiasmierten“, welche sich „so weit zu begeistern vermag, daß diese Begeisterung sie über die engen Schranken der Verhältnisse oder bloßer Vorurteile hinwegtrüge [und so] die ganze Fülle ihres Empfindens nicht nur, sondern auch ihre ganze Tatkraft an die Idee der Demokratie und deren Verwirklichung mit Begeisterung dahin[gibt]. “8

Die neue Idee der ,Assoziation" wurde aber auch aufgegriffen in anderen Lagern und Kreisen: So wollte Johann Hinrich Wichern mit seinem Projekt der „christlichen Assoziation" das Gefälle von Gebern und Nehmern aufheben in ein moderneres Verständnis aktiver Nächstenliebe als solidarischer Partnerschaft.

\section{4. „Vereinswesen“ und „Soziale Verwaltung“: „Wachsende Staatstätigkeit" und subsidiäre Solidarität}

Zum Verhältnis von ,Vereinswesen` und ,Verwaltung ' verdanken wir dem Autor der "Geschichte der sozialen Bewegungen", dem frühen Soziologen Lorenz von Stein auch eine 10-bändige „Verwaltungslehre". In einem eigenen Band „Das Vereinswesen" ${ }^{9}$ würdigte Stein die durch Selbstverwaltung und Ehrenamt geprägten neuen Assoziations- und Organisationsformen bürgerlicher Autonomie (wie Vereine, Stiftungen, Genossenschaften) als Träger öffentlicher Aufgaben und somit als Organe öffentlichen Lebens. Deren Legitimation war nicht staatliche Delegation, sondern das durch Vereinsrecht, also durch Verfahren legalisierte Mandat der sich selbst organisierenden Interessenten.

Die Einbindung sozialer Bewegung in gesellschaftliche Institutionen zeigt die Gewerkschaftsbewegung, aber auch die von Ferdinand Lassalle vertretene Strategie, die sozialrevolutionäre Arbeiterbewegung in eine sozialdemokratische Arbeiterpartei zu überführen. Mit der Transformation der sozialen Bewegungen in die institutionalisierte Form moderner Parteien und Verbände richtet sich die Organisation gesellschaftlicher Interessen nicht mehr gegen das etablierte System, sondern eher auf systemimmanent regulierten Interessenausgleich.

\footnotetext{
8 Frauen-Zeitung, 26. Januar 1850, in: Otto 1991, S. 149.

9 Stein 1869.
} 
Auch das durch die industrielle Dynamik unter Druck kommende ,alte Handwerk“ fand in seiner Existenzangst mit dem durch Schulze-Delitzsch angebotenen Modell der wirtschaftlichen Genossenschaft eine Möglichkeit, durch Selbst-Hilfe zu überleben.

Gemeinsam ist beiden Strategien die Überzeugung eines gesellschaftlichen Bedingungs- und Wirkungszusammenhanges zwischen wirtschaftlicher Sicherheit, gesellschaftlicher Freiheit und politischer Teilhabe. ${ }^{10}$

Wie die Durchsetzung wohlfahrtsstaatlicher Modernität die Relationen von „Staat“ und "Gesellschaft" verschieben mußte, spiegelt das „Gesetz der wachsenden Ausdehnung der öffentlichen bzw. der Staatsthätigkeiten". ".1" Dies bezog sich nicht nur auf die mit neuen öffentlichen Aufgaben steigenden Staatsausgaben, sondern zugleich auch auf eine neue Qualität öffentlicher Macht. Durch die Angewiesenheit auf öffentliche Unterstützung entstanden neue Abhängigkeiten. Bürgerlicher Gemeinsinn mußte also unter den Bedingungen sozialstaatlicher Intervention neu definiert werden. So sollte „wachsende Staatstätigkeit" ihr Gegengewicht finden in institutioneller Aufwertung von Selbstverwaltung und Ehrenamt, von Solidarität und Subsidiarität: Gegenüber der dann im Policy-Feld der „Cultur- und Wohlfahrtszwecke“ drohenden „Gefahr des Centralismus“ galt es, „durch Decentralisation, namentlich in Richtung vom Staat [...] bis zu den Gemeinden hin, und durch Selbstregierung und Ehrenamtssystem, ferner durch Erleichterung und Begünstigung der Gemeinwirtschaften, des Vereinswesens, der Veranstaltungen des caritativen Systems u.s.w. [...] gewissen Gefahren (jener centralistischen Richtung) möglichst zu steuern." 12

Diese frühe Einsicht, daß ,wachsender Staatstätigkeit" nur ,gegenzusteuern“ ist, wenn die Netze wohlfahrtsstaatlicher Hilfen, Halte und Stützen auf sozialer Nähe gründen, führte in Deutschland zu einer „Wohlfahrtskultur", in der Solidarität über das Prinzip der Subsidiarität gebunden ist an den Vorrang sozialer Nähe: sozialräumliche Nähe (Kommunalität als Basis sozialer Selbstverwaltung), kulturelle Nähe gemeinsamen Sinns (Subsidiarität in Kooperation mit wertpluralen ,freien Trägern') und die berufliche Kompetenz, in der helfenden Beziehung zwischen Nähe und Distanz zu balancieren (Professionalität des helfenden Handelns). Zunehmend Bedeutung gewann soziale Nähe schließlich auf der Basis von Selbstbetroffenheit und Mitbetroffenheit (Solidarität als Steuerungsmedium).

\section{Kulturen des Helfens: „Für-Sorge“ und „Selbst-Steuerung“}

Die Frage nach den Kulturen des Helfens verweist auf die Leitbilder einer professionell organisierten und zugleich konfessionell motivierten Solidarität. Dabei wird als Problem bewußt, daß fremde Hilfe, gerade wenn sie - in gut gemeinter Professionalität ihren Adressaten all ihre "Sorge" durch „Für-Sorge" abnehmen will, in die Paradoxie treibt, daß Fremd-Hilfe das eigene "Selbst" zu entwerten droht. Wer fremden Dienst in Anspruch nimmt, unterwirft sich sozialer Kontrolle und wird in seiner Angewiesenheit

10 Zur Geschichte des Assoziationsdiskurses vgl. Pankoke 2000.

1 Wagner 1879, S. 304.

12 Ebd., S. 312. 
auf fremde Hilfe abhängig vom Helfer. So droht im Zuge von Modernisierungsprozessen der persönlich beherrschte Raum zu schrumpfen, während der Verhängnisraum systemischer Abhängigkeiten sich weitet.

Modernisierungskritik verweist dazu auf den Bedeutungswandel sozialer "Sorge", wie es der Philosoph Martin Heidegger in "Sein und Zeit" reflektiert hat: Kritisiert wurde, daß eine von staatlicher Daseinsvorsorge programmierte, organisierte und professionalisierte Fürsorge und Vorsorge die versorgten Menschen nicht nur in ihren „Sorgen“ entlasten will, sondern daß mit der Abwälzung „eigener" Sorgen an Systeme „fremder" Hilfen die natürlichen Netze des Alltags ihre lebenspraktische Bedeutung verlieren. „Entlastung“" müsse in "Entfremdung" umschlagen, wenn fremde Hilfen „dem Anderen die ,Sorge" gleichsam abnehmen und im Besorgen sich an seine Stelle setzen, für ihn einspringen. Diese Fürsorge übernimmt das, was zu besorgen ist, für den Anderen. Dieser wird dabei aus seiner Stelle geworfen, er tritt zurück, um nachträglich das Besorgte als fertig Verfügbares zu übernehmen, bzw. sich ganz davon zu entlasten. In solcher Fürsorge kann der Andere zum Abhängigen und Beherrschten werden, mag diese Herrschaft auch eine stillschweigende sein und dem Beherrschten verborgen bleiben. " 13

Auf diese Entwertung eigener Sorge durch fremde Hilfe antworten Konzepte einer „Hilfe zur Selbsthilfe“, also einer „Fürsorge, die für den Anderen nicht so sehr einspringt, als daß sie ihm in seinem existenziellen Seinkönnen vorausspringt, nicht um ihm die ,Sorge ${ }^{\natural}$ abzunehmen, sondern erst eigentlich als solche zurückzugeben. Diese Fürsorge [...] verhilft dem Anderen dazu, in seiner Sorge sich durchsichtig und für sie frei zu werden. "14

„Dem Menschen seine Sorge zurückzugeben" wäre zynisch, würde dies nur einen Rückzug der sozialstaatlichen Institutionen aus der Leistungsverantwortung bestätigen und rechtfertigen. Hilfe zur Selbsthilfe wäre vielmehr zu verbinden mit einer bewußten Förderung der heute neu geforderten Fähigkeit, dem Ausfall sozialer Nähe durch den Aufbau sozialer Netze gegenzusteuern.

\subsection{Aspektstrukturen und Relationsfiguren:, Verwaltete Welt ${ }^{6}$, , betreuter Mensch', , aktive Gesellschaft ${ }^{\circ}$}

In den unterschiedlichen Aspektstrukturen der Sprachen des Helfens spiegeln sich wandelnde Relationsfiguren der helfenden Beziehung und zugleich Wechsel im Umgang mit Potentialen der Problembearbeitung:

„Verwaltete Welt": Diese Aspektstruktur, in der das Individuum sich nur noch erleben kann als passives ,Objekt' sozialer Versorgung ist bereits an der Sprachhaltung unserer alltäglichen Selbstbeschreibung und Selbstverständigung zu erkennen. Es ist eine Sprache, die der Sprachkritiker Karl Korn als die „Sprache der verwalteten Welt ${ }^{\text {* }}$ kritisiert hat. Typisch für diese Sprache der Verwalter ist ihre transitive Aspektstruktur, die den Menschen zum Objekt perfekt verwalteter Sachzwänge macht: In dieser ,Sprache der verwalteten Welt" wird unsere Perspektive ausgerichtet über das Präfix ,ver-",

\footnotetext{
${ }^{13}$ Heidegger 1986, S. 122.

${ }^{14}$ Ebd.
} 
in dem das perfektionistische „per-“ von lat. ,per-fectus" steckt. Über diese Sprachhaltung objektiviert sich ,der Mensch im Akkusativ": Wer nicht mehr auf sich selbst vertrauen will und deshalb umso rat- und hilfloser wird, wird dann zum „Fall“, zum Objekt einer immer perfekteren Verwaltung, Versorgung, Verfügung. ${ }^{15}$

„Betreuter Mensch": Eine ganz andere Relation sucht die helfende Beziehung über das weiche „be-“, worin die Dativ-Präposition des „bei-“ steckt: „Betreuen“, „,beraten“, „begleiten“, „begegnen“ bedeutet dann die soziale Relation bei einem sein mit Treue, mit Rat, mit Geleit, als Gegenüber. Hier entwickelt sich aktiver Gemeinsinn über die große Tradition eines verbandsmässig organisierten Altruismus und Solidarismus. Doch dessen Hilfen stoßen an kritische Grenzen, wenn im fürsorglichen Beziehungsfeld eines Dativ-Objektes der Hilflose letztlich noch nicht die moderne Freiheit des Selbst erfahren und erlernen kann.

,Aktive Gesellschaft": Eine ganz andere Aspektstruktur als die Objekt-Beziehung zu Menschen im Akkusativ oder im Dativ bevorzugt die Sprache des aktiven "Selbst". Hier stellt sich das "Ich" (oder auch das kooperative „Wir") selbstbewußt in die Position des Subjekts von autonomen Prozessen des Er-fahrens, Er-lebens, Er-arbeitens, Erfindens, Er-öffnens. Diese initiierende und aktivierende Vorsilbe „er-“ läßt sich dann oft mit dem Adverb „selbst" verbinden: selbst erfahren, selbst erarbeiten, selbst erlernen.

\section{Vereinskulturen: Honoratiorenpolitik und Selbstorganisation}

Heute wird die ,Krise des Ehrenamtes` in den Feldern gesellschaftlicher Selbstorganisation und öffentlicher Selbstverwaltung zur praktischen Frage nach neuen Wegen der Personal- und Organisationsentwicklung. Dazu wird die deutsche Vereins-Verfassung in ihren besonderen Prägungen, Förderungen wie Hemmungen eines durch Gemeinsinn bewegten bürgerschaftlichen Engagements zu beachten sein.

Im Verein bestätigt sich die Ehre von Vorstand und Präsidium über die ehrenvolle Wahl durch die Mitglieder. Der Vereins-Vorsitzende versteht sich so ,mit Recht" als erwählter Repräsentant seines Vereins. Dies erfordert allerdings besondere Führungsqualitäten, um die „Ehre des Amtes“ über das Vertrauen der Mitglieder zu verdienen. Um Vertrauen muß man werben. Dazu gehört zunächst die soziale Kompetenz eines „verbindlichen“ Umgangs. Zugleich muß ehrenamtliche Vereinsrepräsentation fähig sein, sich auf die Interessen und Wünsche der Mitglieder einzulassen. Vereinsführung muß zwischen unterschiedlichen Interessen ausgleichen und im mikropolitischen Kleinkrieg um Organisationsmacht auf den jeweils erfolgreichen Trend setzen können.

Die repräsentative Führungskultur des ehrenamtlichen Vorstands fordert also die Bereitschaft, sich selbst von den Strömungen und Neigungen der Mitglieder abhängig zu machen, um diese dann integrativ zur Sprache und zur Wirkung zu bringen. Wenn so die ,innere Umwelt' des ,Vereins' ihre repräsentative Vertretung findet, bedeutet das aber auch umgekehrt, daß der Repräsentant sich für die Repräsentierten selbst kontrol-

15 Vgl. Pankoke 1966. 
lierbar macht. Gerade im ,Dritten Sektor' selbstorganisierten Engagements müssen sich ,Führungskräfte ${ }^{`}$ auf die, inneren Kräfte` ihrer Mitglieder kommunikativ einlassen, also ihre Sprache sprechen, ihre Werte teilen. In einer permanenten Pflege der Mitgliedschaft durch vertrauensbildende Maßnahmen sind die besonderen Motive und Interessen, Emotionen und Energien der Basis anzuerkennen und anzunehmen.

Die Begabungen eines erfolgreichen ,Vereinsmenschen liegen in seinem Sinn für Gemeinschaft. Wer ,vor-steht“, muß im Umgang mit Mitmenschen, insbesondere mit Vereins-Mitgliedern ,leutselig' sein, also Interesse zeigen für das je Eigene des Anderen. Erwartet wird gemeinschaftsfähige Verbindlichkeit und Vermittlungsfähigkeit. Das gilt nicht nur für die selbstgenügsame Gemütlichkeit altdeutscher, Vereinsmeierei ${ }^{\text {. }}$

Vermittlungsfähigkeit muß sich heute neu bewähren unter den Bedingungen einer hochindividualisierten ,Erlebnisgesellschaft'. So sind die traditionell im Vereinsleben geprägten Kompetenzen neu gefordert: als Fähigkeit der verbindlichen Gewinnung und Einbindung von Mitgliedern, als Motivierung und Aktivierung von Ehrenamtlichen, aber auch als Fähigkeit der mikropolitischen Vermittlung unterschiedlich gelagerter Interessen, etwa im Spannungsfeld zwischen Mitläufern und Mitstreitern, zwischen Haupt- und Ehrenamtlichen, zwischen edlen Stiftern und ihren strategisch operierenden Managern. Überall gilt es, Kompromisse zu finden und Konsens zu erarbeiten. Gefordert sind Führungsstile des wechselseitigen Verstehens und (Ver)handelns - oder um es soziologisch zu formulieren: es geht um kommunikative Kompetenzen der ,integrativen" oder auch ,,konsensuellen Führung“"

„Ehre“" im Verein bedeutet für die repräsentativen Ehrenämter, sich, in der Mitte" (frz. ,milieu') von Gleichgesinnten und im Medium von Achtung und Anerkennung tragen (und wählen!) zu lassen. Aber im traditionellen Vereinswesen und seiner ehrenamtlichen Honoratiorenpolitik wird auch kritisiert, daß im Vereinsleben für Mitgliederpflege, Repräsentationskultur und Personenkult allzu viel Zeit, Geld und Kraft verausgabt werde, was im Sinne der Vereinsziele besser ,nach außen" wirksam werden sollte. Dieser Kritik ist allerdings entgegenzuhalten, daß nur durch hohen organisationskulturellen Aufwand das immer knappere und kostbarere ehrenamtliche Engagement gewonnen, gepflegt und gebunden werden kann. Wenn dann aber der Verein sich mehr um sich selbst dreht als um die Herausforderungen der Umwelt, blockiert solch introvertierte Selbstgenügsamkeit des traditionellen Vereinslebens den (inter-) aktiven Bezug auf die Probleme und Potentiale der sozialen Umwelten.

Wie die Thesen zum ,funktionalen Dilettantismus" herausstellen, konnten gerade die ,in Ehren ergrauten' Funktionsträger, die „Grauen Löwen" in ihrem langen Vereinsleben Beziehungsreichtum und Vertrauenskapital so sehr an ihre Person binden, daß diese ,starke Kultur' gemeinschaftlicher Integration den Mangel an Innovationskraft kompensieren kann. Bei langjährig aktiven Honoratioren und Funktionären stabilisiert der ,Stallgeruch“ „,unter dem weiten Mantel der Solidarität“ eine Beziehungspflege, die oft als „Filz-Management" wirksam wird. Aber auch die im vereinsinternen Kleinkrieg in ihrem mikropolitischen Machtinstinkt geschärften ,Schlauen Füchse“ müssen fachliche Defizite managerialer Kompetenz kompensieren. Sie setzen dabei auf cleveres Taktieren mit dem (mikro-)politischen Potential vereinsinterner und -externer Machtquellen. ${ }^{16}$

16 Vgl. Seibel 1992, S. 105 f. 
Beide Typen können also jeweils Mängel in managerialer Professionalität durch ihre hoch personalisierten Führungsstärken ausgleichen oder aushalten, nämlich durch den strategischen Einsatz von Vertrauenskapital und Machtbeziehungen. Durch diese Abhängigkeit der Spitze von der Basis können dann aber auch die Mitglieder auf ihre Vereinspolitiker Einfluß nehmen.

Die ,starke Kultur ${ }^{6}$ einer das Vereinsleben prägenden konsensuellen Repräsentation der "Stärke des Wir" zeigt allerdings auch Grenzen und Schatten. Auf dem Wege zur innovativen Steuerung bindet das Konsensmodell die Vereinsaktivitäten zu stark an die Mitgliederakzeptanz, deren Mehrheit um die eigene ,Mitte ${ }^{6}$ kreist. Um die riskante Komplexität der aus turbulenter Umwelt auf einen Verein zukommenden Probleme und Potentiale zu meiden, setzt sich im Verein eher eine ,mittlere Linie' durch, wie es auch die ,aus der Mitte' der Mitglieder kommenden Ehrenämter einst zu repräsentieren wußten. Es ist ein „Konservatismus aus Komplexität“ (Luhmann): Traditionspflege, Stabilität und Kontinuität, Ehrung des Alten und der Alten; kurzum: „Keine Experimente“.

\section{1 Ökonomie der Ehre: Leistung, Aufwand und Anreiz}

Für Organisationen im Dritten Sektor stellt sich die Frage, welche Anreiz- und Belohnungssysteme die Entwicklung solidarischen Engagements aktivieren, aber auch blokkieren können. Für ehrenamtliche Führungseliten größerer Vereine und Verbände ist oft der Aufwand durch , angemessene' Aufwandsentschädigung reguliert. Andere sachliche Gratifikationen bieten sich an über die Nutzung von institutionellen und infrastrukturellen Möglichkeiten organisatorischer Hilfen. Noch umstritten als Anerkennung ehrenamtlichen Engagements sind steuerrechtliche, versicherungs- und versorgungsrechtliche Konzepte der Würdigung von „Dienst"-Zeiten.

Das klassische Muster war bis heute, daß der/die Ehrenamtliche seine/ihre Ehre begründet in der Freiheit von materiellen Sorgen: Traditionell ermöglichte dies das gesicherte Einkommen feudaler oder ökonomischer Eliten. Typischer werden heute Lebenspläne, in denen ein verdienter Ruhestand für ehrenamtliches Engagement freie Zeiten anbietet, oder, daß neben bezahlter Berufsarbeit sich Zeit für freies Engagement einräumen läßt. Nicht selten sucht auch eine durch die Routinen von Beruf und Betrieb verweigerte Professionalität im ehrenamtlichen Engagement professionelle Selbsterfahrung und Selbstbestätigung. Oft biete dann gerade das Ehrenamt die Erfahrung und Entfaltung einer „Professionalisierung nach Dienstschlu $\beta^{\text {“. }}$.

Demgegenüber haben wir heute in der Krise der Arbeitsgesellschaft neben diesen klassischen Mustern ökonomisch gesicherter, freier Zeit' auch die ,leere Zeit' ungewollter Arbeitslosigkeit. Dann wird das Ehrenamt im Verein oder die Gruppenarbeit der Selbsthilfe auch zur Geldfrage. So ist auch über Geld im ,Dritt-Sektor' neu nachzudenken, gerade wenn die ehrenamtliche Arbeit nicht von beruflichem Arbeitseinkommen oder anderen Vermögens- und Einkommensquellen gedeckt ist. Neuere Konzepte einer Institutionalisierung des Ehrenamtes als „Bürgerarbeit“ bedeuten allerdings auch ,neue 
Risiken“, in den „Krisen der Arbeitsgesellschaft" die mögliche „Beschäftigungswirksamkeit" des Dritten Sektors zu verfehlen und zu verdrängen. ${ }^{17}$

Zur Würdigung des letztlich unbezahlbaren sozialen Engagements zählt aber nicht nur finanzielle Entlastung. Wichtiger Motivationsfaktor ist gewiß auch ein institutionelles Emstnehmen der ,freien Kräfte" durch einen organisatorischen Rahmen und kommunikativen Kontext, welcher dem Engagement Autonomie eröffnet.

\subsection{Freies Engagement: Grenzgänger zwischen Dienst und Selbstbezug}

Die traditionelle Verbindung von Ehre und Amt gründete in der Bereitschaft, die soziale Mitte einer Gemeinschaft oder einer Gemeinde durch die freie Übernahme von Gemeinschaftsaufgaben und Vertretungspflichten zu repräsentieren. Die Kulte der Ehre, die diese Mitte symbolisieren und repräsentieren, bezeichnen wir als Repräsentationskulturen: Wer aus der Mitte seines in sich geschlossenen Lebenskreises (frz. milieu) kommt, dem wird es zur Pflicht und zur Ehre, seinen Platz in dieser Mítte einzunehmen. Das heute geforderte „neue Ehrenamt“ bleibt hingegen nicht mehr in sicherer Mitte, sondern wagt sich hinaus an die Ränder der etablierten Normalität und Modernität. In einer Gesellschaft, die sich selbst definiert über den „Verlust der Mitte", ist auch das gemeinsinnige Engagement kaum noch durch einen verallgemeinerbaren Sinn getragen. Dies treibt soziales Engagement heute heraus aus der sicheren Mitte des normalen Alltags in Risikofelder, Tabuzonen und Konflikträume. Jenseits aller alltäglichen Vertrautheit aber wartet kaum noch allgemeine Anerkennung, sondern Unverständnis und Mißtrauen.

Mit dem Wechsel von „Ehre“ zum „Engagement" wechselt der/die Engagierte aus der Mitte seines normalen Alltags an dessen Grenzen: Er wird zum „Grenzgänger“, zum „Moderator" und „Mediator", oft auch zum „Partisanen“" und „Piraten“ in den Risikozonen und Konfliktfeldern, die im Alltag nur zu gerne verdrängt werden. Grenzfragen konfrontieren die Routinen des Alltags mit Konflikten und Krisen, mit Devianz und Anomie, mit Sucht und Siechtum, Alter und Tod - alles Probleme, die der moderne Mensch nur zu gern aus seinem Alltag abschieben will. Vielfach werden solche „Grenzfragen" des Lebens auch zum Thema neuer Formen gruppendynamischer Selbsthilfe und Selbsterfahrung, was gleichermaßen anzeigt, daß viele Probleme nicht nur im Alltag, sondern auch im offiziellen und professionellen Hilfesystem gerne ausgegrenzt und abgeschoben werden. Gefordert ist dann Rat und Hilfe durch freie „Grenzgänger", die sich den „Grenzfragen" personaler und sozialer Lebenskrisen aktiv und solidarisch stellen. Dies gilt für kritische Felder der Suchtkrankenhilfe, der Telefonseelsorge, der Konfliktberatung, der Gefährdetenhilfe, der AIDS-Hilfe, der Solidarhilfe mit psychisch Kranken und geistig Behinderten, der Hilfe für Eltern von Sorgenkindern oder auch der Hilfe in Grenzfragen des Lebens bei der Betreuung altersschwacher Menschen oder in der Hospizbewegung.

All diese Felder am Rande gelten kaum noch als „ehrenhaft" im Sinne der älteren Repräsentationskultur traditioneller Honoratioren. Wer in den neuen Feldern aktiv und

\footnotetext{
17 Erlinghagen 2001.
} 
initiativ wird, dem geht es nicht mehr um den Glanz der ,Mitte', vielmehr müssen die freien Helfer eigene Berührungsängste überwinden und sich oft auch verstricken lassen in Schmutz, Schuld und Leid kritischer Problemfelder und personaler Lebenskrisen. All dies fordert eher ein Engagement, das hinaustreibt aus der sicheren Mitte der Normalität an die Ränder moderner Risikogesellschaft - bis an die Grenze ihrer Tabuzonen und Konfliktlagen. Das neue Engagement fordert auch ein neues Management der Steuerung und Selbststeuerung. ${ }^{18}$

Diese Differenzierung von „alter Ehre" und „neuem Engagement" ist auch zu beachten im Blick auf einen auch in Deutschland diskutierten „Schwund des Sozialkapitals". ${ }^{19} \mathrm{Zu}$ unterscheiden wären unterschiedliche Ebenen der „Assoziabilität" im Spektrum von gesamtgesellschaftlich organisierten Großverbänden bis hin zur situativen Initiative. Dabei zeigen sich zugleich unterschiedliche Orientierungen von Ehre und Engagement. $\mathrm{Zu}$ unterscheiden wäre etwa die Traditionalität von bindenden Werten, die Rationalität gemeinsamer Interessen oder die Reflexivität der gemeinsamen Sinnkonstruktion gemeinsinnigen Engagements:

Traditionalität als Bindemittel scheint sich heute zu lockern, vor allem in den großen Traditionsverbänden, wenn sich hier die Mitgliedschaft in der Zahlung von Mitgliedsbeiträgen zu erschöpfen scheint und dies die Mitglieder von weiterem und aktiverem Engagement entlastet. Vor diesem Problem stehen heute auch Parteien, Gewerkschaften, Kirchen.

Rationalität des Mitgliedschaftsinteresses wird hingegen angestrebt, wenn Verbände ihre Mitglieder für sich zu interessieren suchen, indem die Mitgliedschaft kalkulierbare, oft ,geldwerte ${ }^{c}$ Vorteile und Vorzüge (etwa durch ,günstige' Erlebnis-, Bildungs-, Beratungsangebote) versprechen will. Die Vorteile der Mitgliedschaft liegen zumeist allerdings weniger in finanziellen Vorteilen als in oft viel interessanteren Chancen für soziales oder kulturelles Kapital, wobei dessen Produktion mit gleichzeitiger Konsumtion verbunden ist.

Reflexivität der Sinnkonstruktion sieht sich gefordert und gefördert, wenn das soziale Kapital des selbstorganisierten Engagements anderen zugute kommt, denen man sich solidarpartnerschaftlich verbunden weis, etwa Hilfsbedürftigen der eigenen Gesellschaft, oder auch in globaler Solidarität zur ,Dritten Welt'. Dabei geht es gewiß weniger um die traditionelle Dauer-Bindung von Ehre und Amt, wohl aber um aktive, oft spontane Antworten auf Kritik und Krise in der eher offenen Form von Projekt, Initiative und Aktion. In der (inter-) aktiven Begegnung mit Anderen und Fremden, wie auch in der gemeinsamen Selbstverständigung selbstorganisierten Engagements eröffnen sich für die aktiv Engagierten aktive Lernfelder der Grenzerfahrung, der Kompetenzerfahrung und der Selbsterfahrung. In der Wende „vom Dienst zum Selbstbezug ${ }^{620}$ gewinnt diese eher offene Form situativen Engagements gerade für jüngere, intellektuell gebildete und selbstbewußt ,moderne' Menschen offensichtlich neue Attraktivität.

Entsprechend wird, wie empirische Studien bestätigen, den eher informellen Formen sozialer Vernetzung gegenüber den formalisierteren, konventionellen und traditionellen

\footnotetext{
18 Siehe dazu Pankoke 2001.

${ }_{19} \mathrm{Vgl}$. Offe/Fuchs 2001.

20 Siehe dazu Jakob 1993.
} 
Mustern der Vorzug gegeben. ${ }^{21}$ Nach den von Offe und Fuchs ausgewerteten Befunden, ist auf der Ebene der klassischen „Massen-Organisationen“ die Mitgliedschaft und ihre Beitragsbereitschaft deutlich rückläufig, während andere Formen aktiven Engagements, welche die Handlungskompetenz, die Lernbereitschaft und die Verantwortungsfreude der Aktiven sehr konkret fordern und dann auch Wirkungen des freien Einsatzes von Ressourcen, Kompetenz und Engagement unmittelbarer spürbar machen, sich wachsender Nachfrage erfreuen. Auf eine Formel verdichtet, verliert die bloße, eher passiv mitlaufende Mitgliedschaft in einem Großverband an Interesse. Die Bereitschaft zu aktivem Engagement steigt jedoch, wenn - außerhalb, gewiß aber auch innerhalb der traditionellen Großverbände - ein engagierter Gemeinsinn in der sozialen Aktivität von Netzwerken und Lernprozessen seine gesellschaftliche Form finden kann.

\section{Neue Praxis: „Empowerment“ durch ,Solidar-Partnerschaft“}

In der professionellen Selbstverständigung sozialer Arbeit kommt es zu einem strategischen Blick- und Führungswechsel: so sollen die Potentiale selbstbewußter Autonomie nicht nur eingefordert werden für die Kompetenz und das Engagement des Helfers; auch der von Problemen der Rat- und Hilflosigkeit Betroffene will als Subjekt der Erfahrung und Bearbeitung seiner Probleme erst genommen werden. Aus Objekten fremder Hilfe werden dann Subjekte eigener Sorge - und aus Patienten werden Partner einer gemeinsamen Problembearbeitung.

Während die traditionellen Konzepte einer fürsorglichen „Hilfe zur Selbsthilfe“ die Wiederherstellung der Normalitätsstandards einer selbständigen Lebensführung subsidiär stützen sollen, wollen neue Konzepte sozialer Praxis unter der Programmformel „Empowerment" gerade solche Kräfte stabilisieren, welche den Rahmen ihres Alltags sprengen. Dies bedeutet zugleich einen Perspektiven- ja Paradigmenwechsel vom reaktiven FallBezug zur Feldorientierung einer aktiven und aktivierenden Sozial- und Gemeinwesenarbeit. Empowerment setzt auf die sozialen Stärken im „Feld“, gerade auch dann, wenn wie bei schwerem „Fall“ der Alleingang einem Problem nicht mehr gewachsen ist.

\section{Neue Arbeit: „Lokale Ökonomie“ und „Soziales Kapital“}

In heute akuten Modernisierungskrisen (und Modernisierungsschwellen) der Arbeitsgesellschaft könnten ,intermediäre" Konfigurationen solidarischer Partnerschaft neue Perspektiven der Beschäftigung eröffnen: zwischen einen im dritten Sektor aktivierbaren Verbund von „lokaler Ökonomie" und „sozialem Kapital“ und dem ökonomischen System des Marktes bzw. dem politisch-administrativen System.

Als Basis und Rahmen für einen, zweiten Arbeitsmarkt" bietet das gesellschaftspolitische Engagement von freien Trägern und freien Initiativen - etwa über genossenschaftliche Selbsthilfe und Selbstorganisation - neue Chancen für assoziative Beschäf-

${ }^{21} \mathrm{Vgl}$. Offe/Fuchs 2001, S. 503 f. 
tigungsinitiativen ,jenseits von Markt und Staat". Damit gewinnt der ,Dritte Sektor ${ }^{\text {c als }}$ institutioneller Rahmen für einen „Zweiten Arbeitsmarkt“ aufs Neue praktisches Interesse. Dazu gibt es heute überall in Europa soziale Experimente am Rande der Arbeitsgesellschaft. Die Programme und Probleme neuer Wege in die aktive Gesellschaft markiert die Studie des italienischen Soziologen Enrico Taliani (1996) Marginalità sociale e nuova progettualità, sinngemäß übersetzt Soziale Randständigkeit und Projekte des Aufbruchs. „Progettualità“ - in dieser schwer ins Deutsche übersetzbaren Programmformel steckt nicht nur das modisch gewordene ,projektive Denken“" von ProjektOrientierung und Projekt-Management, sondern auch die ,progressive" Ausrichtung auf die fortschrittliche Dynamik „sozialer Bewegung “. ${ }^{22}$

\section{Neue Steuerung: Qualitätsentwicklung und soziale Selbststeuerung}

Der Perspektiven- und Führungswechsel von regulativer Rationalität zur Reflexivität neuer Steuerung richtet soziologisches Interesse auf die Zusammenhänge zwischen Akteursprofilen selbstorganisierten Engagements und den Organisationsstrukturen und Kommunikationskulturen , gemeinsinniger' Organisationen:

Transitive Konzepte einer auf die geradlinige Durchsetzung zentraler Ziele fixierten Führung scheinen nun ablösbar durch eine reflexive Steuerung - etwa im Modus von Supervision oder auch Evaluation. Die Steuerungsinstanzen greifen dann in die laufenden Prozesse nicht ein durch Befehlskommunikation, wohl aber durch Mitteilung von Beobachtungen, die für den Handelnden sonst im blinden Fleck liegen können. Doch Supervision kann allenfalls eine Revision der Handlungsrichtung zu bedenken geben; die Kurskorrektur in der ,Optik' des Handelns und Wertens muß dann jedoch aus der Einsicht des Akteurs selbst kommen. Es geht nun darum, hier das Medium zu erkennen, über das eine, intelligente Organisation` im Wechselspiel von Umweltsensibilität und Selbstbeobachtung lernen kann, sich selbst zu steuern. Dies gilt auch für die Umstellung von rationalistischen Erfolgs- und Kostenkontrollen herkömmlicher Revision auf neue Verfahren der Supervision, der Evaluation und der Selbst-Evaluation.

Grundsätzlich lassen sich drei Strategien der Reaktion auf Umweltveränderungen unterscheiden: Selbstgenügsamkeit, Selbstregulierung, Selbststeuerung: ${ }^{23}$

Selbstgenügsamkeit: Stabilität durch soziale Schließung. Man läßt alles beim Alten. Problemdruck, der aus der Umwelt auf ein System organisierter Hilfe zukommt, bleibt unbeantwortet. Doch das selbstgenügsame „Einigeln“ in wertrationale Geschlossenheit geriet unter Modernisierungsdruck. Nicht zufällig kommt es heute in den Leitbildprozessen der wertorientierten Traditionsverbände des „Dritten Sektors“ zu selbstkritischer Selbstverständigung. Im Interesse der Produkt- und Prozeßqualität sozialer Dienste

${ }^{22}$ Zum „dritten Sektor“ als „dritter Kraft“ vgl. Strachwitz 1998; zum „Zweiten Arbeitsmarkt“ vgl. Trube 1997.

${ }^{23} \mathrm{Vgl}$. Pankoke/Nokielski/Beine 1975. 
werden tradierte Organisationsprinzipien und Relationsmuster neu zur Diskussion und zur Disposition gestellt. Damit wird die ältere Selbstgenügsamkeit verabschiedet.

Selbstregulierung: Effizientes und flexibles Qualitätsmanagement. Aufgeschlossen für Veränderungen in der Umwelt, versucht das Management jede Lücke zu füllen und jede Chance zu nutzen. Gefordert wird eine permanente Flexibilität des Sich-Anpassens an die Opportunitäten der Umwelt. Zur Selbstregulierung gehört für Dritt-SektorOrganisationen die Bereitschaft, sich den Systemzwängen des modernisierten Sozialstaats wie auch dem Rationalisierungsdruck dynamischer Sozialmärkte anzupassen. Auch in Vereinen und Verbänden signalisiert der Ruf nach Sozialmanagement neue Strategien der Rationalisierung und Regulierung. Dabei kommt ein an Rationalisierungsstrategien des wirtschaftlichen Unternehmens orientiertes Qualitätsmanagement in Spannung zur wertrationalen Identität der traditionellen Träger.

Der neue Konkurrenzdruck des Aushandelns zwischen Leistungserbringern und Kostenträgern „verlangt Transparenz von Kosten, Leistungen und Qualitäten“. Das fordert auch ein neues Verhältnis zu den Leistungsnehmern, die nun auch die Perspektive wechseln, indem Klienten zu „Kunden“, Patienten zu „Partnern“" werden. Für ein sich als Qualitätsmanagement verstehendes Sozialmanagement bedeutet dies ein „dynamisches Qualitätsverständnis, das der Stärkung der Kunden in ihrer Verbraucherrolle im Alltag der Dienstleistungserbringung gerecht wird“. ${ }^{24}$

Selbststeuerung: Relationen reflexiver Autonomie. Strategien der ,Selbststeuerung setzen auf die Aktivierung der Potentiale von Spontaneität und Kreativität. Dies fordert allerdings nicht nur anpassungsbereite Flexibilität, sondern die Reflexivität von Lernprozessen, also die „Verantwortlichkeit“ dafür, daß die Antwort auf neue Herausforderungen auch bedeuten muß, sich selbst' zu ändern und zu steuern. Eine entsprechende Personal- und Organisationsentwicklung zielt also auf das Lernen in Netzwerken und die Vernetzung von Lernprozessen. Dabei vollzieht sich „Entwicklung“ immer auch über die „Beteiligung“ der als Betroffene, Verantwortliche, Lernende einbezogenen Subjekte. So kommt es zu Reflexionsprozessen, in denen sich Qualitätsmanagement mit der Profilierung von Organisationskultur und „Corporate Identity“ zu verbinden sucht.

Das Spannungsfeld selbstorganisierten Engagements zwischen Mandat, Mission und Management wird allerdings zum Problem und Politikum, wenn es darum geht, das beanspruchte Mandat im Sinne von Solidar-Partnerschaft zugleich gegenüber dem sozialstaatlichen Gesetzgeber, also dem wohlfahrtstaatlichen Kostenträger, und in einer gesellschaftspolitisch interessierten Öffentlichkeit zu legitimieren.

\section{Neue Horizonte in inter- und supranationalen Transformationen}

Die sozialwissenschaftliche Verbändeforschung verweist im europäischen Vergleich auf die unterschiedlichen Ausprägungen von Zentralismus, Föderalismus und Korporatismus. ${ }^{25}$ Es wird zu untersuchen sein, inwieweit diese Profile in den Transformationsprozessen der Europäischen Integration sich halten, steigern oder verändern können. So

24 Brückers 1999, S. 75.

$25 \mathrm{Vgl}$. Schmid 1996. 
ist gerade bei Modernisierungen des „Dritten Sektors“ der europäische Trend zu beachten und zu verdeutlichen, daß Traditionalität überführt wird in Rationalität und Rationalität sich relativiert im Horizont institutioneller Reflexivität.

So beobachten wir eine Belebung, aber auch eine Veränderung der europäischen Vereinslandschaft:

„Die Vereinsdichte hat sich seit 1960 in Deutschland verdreifacht, in Frankreich und Österreich finden sich ähnliche Zahlen. [...] Wir sind immer häufiger Mitglieder in immer mehr Vereinen, die aber immer weniger die Großvereine oder Gemeinschaften sind, die in ihrer jetzigen Verfassung aus dem industriellen Zeitalter stammen. Die Vereinslandschaft wandelt sich. “26

Möglicherweise kann die Europäisierung aber auch die in den nationalen Wohlfahrtskulturen verfestigten Entwicklungssperren aufbrechen und der Selbstorganisation aktiven Engagements, nicht nur über die neuen Geldquellen des Europäischen Sozialfonds, neue Perspektiven öffnen.

„Europa bietet also die Chance, das auf europäischer Ebene zu erreichen, was staatliche und auch verbandliche Politik und Interessenlagen auf nationaler Ebene verhindern bzw. erschweren. Europa bedeutet in vieler Hinsicht eine Politik der Deregulierung und Entflechtung. [...] So werden in den kommenden Jahren viele Freiräume von und in Europa geschaffen werden, zu Umstrukturierungen, ja Umwälzungen auf nationaler Ebene führen, [...] um somit das zivilgesellschaftliche Fundament einer zukünftigen europäischen Gesellschaft zu bilden."27

Längst weitete sich der Horizont neuer sozialer Bewegungen in die Dimensionen von Weltgesellschaft. Mit der Globalisierung von Massenkommunikation, die kulturelle Impulse weltweit durchschlagen läßt, müssen sich Betroffenheit von systemischer Benachteiligung und solidarische Mitbetroffenheit heute weltweit orientieren, organisieren und aktivieren. Auch hier formieren sich globale Bewegungen im Dritten Sektor als weltweit operierendes Engagement für Frieden, Gerechtigkeit und Bewahrung der Schöpfung. ${ }^{28}$ Gerade die europäische Einigung bietet Fokus und Forum der supranationalen Koordination und Repräsentation gerade der globalisierungskritischen Bewegungen des Dritten Sektors. „Gemeinsinn“ gewinnt mit der europäischen Öffnung neue Horizonte. ${ }^{29}$

\section{Neue Politik: Zur aktiven Öffentlichkeit freien Engagements}

In den heute akuten Modernisierungskrisen und Modernisierungsschwellen gewinnt die bürgergesellschaftliche Aktivierung von ,Civic Culture` neue Aktualität. Im Umbau gesellschaftlicher Strukturen und politischer Kulturen setzen neue Programmformeln einer ,Active Society" auf, selbstaktive Felder", in denen die Bürger als selbstbewußte Akteure und Konstrukteure ihrer Verhältnisse in die Verantwortung gerufen sind. ${ }^{30}$

26 Anheier 1999, S. 20.

${ }^{27}$ Ebd., S. 21.

$28 \mathrm{Vgl}$. Greenpeace, WWF, Amnesty International.

29 Siehe dazu Münch 1999, S. 228.

30 Vgl. Kaufmann 1992. 
Damit gewinnt eine politische Kultur an Gewicht, deren Semantik die Traditionen von „Stiftung" und „Innung“, „Ehrenamt“ und „Vereinswesen“ überführt in die neue Programmatik aktiver „Bürgergesellschaft“. Hier geht es darum, daß Menschen nicht nur bei privaten Sorgen, sondern auch bei öffentlichen Problemen sich selbst und anderen zu helfen wissen.

Im Blick auf den Gemeinsinn bürgergesellschaftlichen Engagements wird also zu fragen sein, welche Verfasstheit öffentlichen Lebens (z.B. Wahlsystem, Partizipationsregeln, intermediäre Instanzen, Vereins- und Stiftungsrecht) einer aktiven Öffentlichkeit förderlich oder hinderlich werden. Institutionelle Rahmungen bürgerschaftlichen Engagements fänden sich etwa im kommunalen Verfassungsrecht, im Planungsrecht, im Vereins- und Stiftungsrecht, in den Verfahren zur Ausgestaltung ordnungspolitischer Prinzipien der Subsidiarität und Pluralität, oder auch in den offenen Foren und runden Tischen öffentlicher Partizipations- und Mediationsprozesse. In all diesen Feldern ,zwischen Markt und Staat' könnten die Organisations- und Legitimationsmuster des ,Dritten Sektors' theoretisches Interesse und praktische Relevanz gewinnen, weil hier der Gemeinsinn von Ehrenamt und ,freiem Engagement ${ }^{6}$ nicht nur moralisch beschworen wird, wie in der moralischen Rhetorik des Kommunitarismus, sondern soziologisch zu beobachten und praktisch zu befördern ist.

„Neue Steuerung" im Rahmen von „Public-Private-Partnership“ bedeutet nicht nur eine durch fiskalische Knappheit diktierte Rationalisierung, sondern auch ein Aktivieren neuer Ressourcen und Potentiale gesellschaftlicher Entwicklung.

In dem Maße wie die Selbstorganisation bürgerschaftlichen Engagements heraustritt aus vereinsmeierlicher Selbstgenügsamkeit und sich in Konstellationen des PublicPrivate-Partnership in öffentlichen Belangen und Aufgaben engagiert, steigen die Erwartungen an die Handlungs-, Lern- und Steuerungsfähigkeit sowohl gegenüber gesellschaftlicher Selbstorganisation wie auch gegenüber dem politisch-administrativen System der kommunalen Ebene.

Selbstaktive Felder sehen sich nun neu gefordert, im Verhältnis zu öffentlicher Macht, öffentlichen Mitteln und öffentlicher Meinung neue Handlungs- und Geschäftsfähigkeit zu entwickeln. Politisch-administrative Systeme müssen im Verhältnis zu den Vereinen neue Muster und Werte der Verständigungsbereitschaft und Verhandlungsfähigkeit entwickeln.

Also kommt es auf beiden Seiten, in den selbstaktiven Feldern bürgerschaftlichen Engagements wie auch im politisch-administrativen System darauf an, wechselseitig Vermittlungsfähigkeiten zu entwickeln. In den selbstaktiven Feldern des Dritten Sektors wird es deshalb wichtig, bei der Übernahme neuer und ungewohnter Angebote die eigene Leistungs- und Lernfähigkeit selbst zu beobachten und zu steuern. Aber auch im politisch-administrativen System sind neue, interaktive, ja intersubjektive Relationsmuster im Verhältnis zu selbstaktiven Feldern bürgerschaftlichen Engagements immer wieder neu zu erlernen. 


\section{Macht-Geld - Sinn: Nachhaltigkeit und Zukunftsfähigkeit}

Als Gegentyp zum traditionellen Vereinsleben entwickeln sich heute neue Aktionen und Initiativen. Hier geht es weniger um die Repräsentation von Ehre als um die Reflexion von Engagement. Bei der Aktivierung von selbst-organisiertem Engagement bewähren sich heute auch die aus der Gemeinwesenarbeit entwickelten Modelle und Methoden des „Community-Organizing": „Community“ muß über professionell gesteuerte Prozesse der Selbst-Organisation neu gestaltet und entwickelt werden. Nach dem Vorbild der amerikanischen ,Gemeinschafts-Stiftung' (Community-Foundation) eröffnen sich zukunftsweisende Ansätze der Institutionalisierung bürgerschaftlichen Engagements. Beispiele geben Bürgerstiftungen, Publikums-Stiftungen oder auch Stadt- und RegionalStiftungen. Über die stiftungsrechtliche Bündelung der aus der Bürgerschaft kommenden Klein- und Zu-Stiftungen, entsteht dann ein Rahmen, in den sich bürgerschaftliches Engagement finanziell wie institutionell konstruktiv einbringen kann. ${ }^{31}$

Auf öffentlich verantwortbare Nachhaltigkeit setzten jene Bürgerstiftungen, die im Rahmen der „Agenda 21 "-Prozesse zur lokalen Umsetzung einer globalen UmweltVerantwortung den Versuch wagen, ,sich aktiv und unter aktiver Beteiligung der Stifter ebenso wie anderer Bürgerinnen und Bürger an der Entwicklung und Durchführung von Projekten zu beteiligen oder diese allein und sogar bewußt als konzeptionelle Alternative zu anderen zu gestalten“, und so „eine nachhaltige Entwicklung unter Einbeziehung ökologischer, sozialer und kultureller Aspekte auf lokaler Ebene in Gang zu setzen" 32 Vielleicht ist es diese institutionalisierte Nachhaltigkeit eines zukunftsweisenden Engagements, welche in einer neuen Bürgerkultur den Konstruktionen der Bürger- und Gemeinschaftsstiftungen ihre besondere öffentliche Verantwortung zuweist.

Bei der Unübersichtlichkeit riskanter Wirkungsketten wird allerdings eine personale Zurechenbarkeit öffentlicher Verantwortung immer schwerer zu identifizieren sein. Personalität als das der Verantwortungsflucht entgegengehaltene Sozialprinzip macht es zur Schlüsselfrage der modernen Gesellschaft, wie gesellschaftliche Akteure sich in die Verantwortung, rufen' lassen und in welchem Horizont sie diesem Ruf antworten.

Heute allerdings wird den modernen Organisationen oft eine höhere Verantwortlichkeit zugesprochen als den einzelnen organisierten Akteuren. Die Frage nach der Nachhaltigkeit institutioneller Verantwortung wird so ihren Horizont weiten müssen auf eine an künftigen Entwicklungen orientierte „Ethik der Zukunftsverantwortung “ - im gemeinsamen Horizont einer, ,Verantwortung für die Zukunft der Menschheit“ ${ }^{33}$

\subsection{Soziales Kapital und reflexive Kultur}

Der Begriff des „Dritten Sektors“ beansprucht programmatische Differenz zu den über ,Macht" und ,Geld ' rationalisierten Leitsystemen der industriellen Moderne: dem über Geldzahlungen kontrollierten Marktsystem und der staatlichen Verwaltung öffentlicher

\footnotetext{
31 Vgl. Feurt 1999.

${ }^{32}$ Strachwitz 2000, S. $114 \mathrm{f}$

33 Kaufmann 1992, S. 112.
} 
Macht. Demgegenüber richtet sich die Frage nach dem ,Sinn' selbstorganisierten Engagements auf soziale Qualitäten, wie sie auf Märkten nicht zu haben sind, aber auch durch öffentliche Macht so nicht zu erzwingen sind. Macht bedeutet dann nicht nur Druckmittel (Pression) der transitiven Durchsetzung fester Zwecke, sondern auch Ermöglichung (Potential) aktiver Kräfte, wie sie sich ökonomischer Rechnung und staatlicher Regelung weitgehend entziehen.

Im Unterschied zum wirtschaftlichen Kapital bezieht sich ,Sozialkapital ${ }^{\star}$ auf den ,menschlichen' Wert der sozialen Bindungen, Beziehungen und Vernetzungen. Dazu sind gewiß auch die klassischen Kapitaltheorien wie deren Kritik lehrreich: die „National-Ökonomie" des Adam Smith, wonach Geld zum Kapital wird, das ,arbeitet" und dadurch wachsen wird - wobei die jeweils privaten Interessen zugleich den „Reichtum der Nationen" befördern. Dagegen eröffnete später Karl Marx seine unter dem Titel „Das Kapital" veröffentlichte „Kritik der politischen Ökonomie" mit dem Hinweis auf den verhängnisvoll blendenden Schein des ,Reichtums einer Gesellschaft [...] als ungeheure Warensammlung ".

Demgegenüber definieren neuere Konstruktionen des ,sozialen Kapitals ${ }^{6}$ in Verbindung mit der Lehre „öffentlicher Güter" eine andere Qualität gesellschaftlichen Reichtums: So erhöht das „soziale Kapital", etwa als „Beziehungsreichtum“, nicht nur die individuelle Lebensqualität, sondern fördert als "öffentliches Gut" zugleich Kräfte der gesellschaftlichen Entwicklung. In der Theorie des „,sozialen Kapitals“ wird dazu unterschieden zwischen dem „informellen Sozialkapital“ (der Beziehungsfähigkeit in den ,natürlichen Netzen“ persönlicher Nähe) und „formal organisiertem Sozialkapital“, wie es in den Netzwerken selbstorganisierter Solidarität , arbeiten' kann. ${ }^{34}$

Von „öffentlichen Gütern“ spricht die Wohlfahrts-Ökonomie in Abgrenzung von allem, was auf Märkten für Geld zu kaufen ist. Den quantitativen Maßen privaten Geldes und privaten Nutzens entziehen sich all jene „Qualitäten des Lebens“ und „Reichtümer der Gesellschaft", deren Verteilung und Vermittlung sich über den Marktmechanismus von Angebot und Nachfrage nicht ,rechnet', deren Konsum sich nicht über konkurrierende Nachfragen regelt und deren Produktion auch nicht über den Preis der Produkte zu finanzieren ist.

Öffentliche Güter werden deshalb zum allgemeinen Nutzen vom politischen Gemeinwesen garantiert, und zwar durch den Einsatz von öffentlicher Macht und öffentlichen Mitteln. Dies gilt etwa für die Regelwerke der rechtlichen und sozialen Sicherung, oder auch für die Infrastrukturen gesellschaftspolitischer Policy-Felder der Umwelt-, Verkehrs-, Sozial-, Bildungs- oder Kultur-Politik. Doch schon im Rückbezug auf Wagners ,Gesetz der wachsenden Staatstätigkeit“" (Wagner 1879: 308-325) wurde deutlich, wie sehr die Kontrollmedien ,Macht' und ,Geld“ an Grenzen stoßen. Für die Qualitäten des Sozialen sind andere Kräfte gefragt: das soziale Kapital selbstorganisierter Solidarität und die kulturelle Reflexivität der Sinnkonstruktionen freien Engagements.

Nach den Befunden von Robert D. Putnam wirkt der vertrauensbildende Effekt, sozialen Kapitals' insbesondere in horizontalen Kooperationen unter Gleichen als Gegen-

${ }^{34}$ Vgl. zur Gemeinsinn-Qualität sozialen Kapitals jetzt Putnam 2001, zur netzwerktheoretischen und transaktionsökonomischen Operationalisierung von sozialen Kapitalien und öffentlichen Gütern Herrmann-Pillath/Lies 2001. 
gewicht zur den vertikalen Asymmetrien von Mächten und Märkten. Dabei haben horizontale Netze nicht nur einen ,internen" Nutzen für die unmittelbar Beteiligten; zu würdigen sind auch die ,externen' oder ,öffentlichen' Effekte von Beziehungsreichtum und Vernetzungsdichte für die soziale, kulturelle, aber auch ökonomische Produktivität einer Gesellschaft. ${ }^{35}$ Zugleich fördert das soziale Kapital produktive Wechselwirkungen Zwischen, privatem Glück' und ,öffentlichem Wohl ${ }^{*}{ }^{36}$

$\mathrm{Da}$ privates Glück zugleich das öffentliche Wohl fördert, womit soziale Aktivität zur aktiven Gesellschaft führen kann und umgekehrt, wird nicht nur von kommunitaristischer Programmatik beschworen, sondern bestätigt sich auch durch empirische Sozialforschung. Dazu erfassen wir das Sozialkapital gemeinschaftlichen und gemeinsinnigen Engagements über drei Indikatoren: „Aufmerksamkeit“, „Vertrauen“ und „Engagement in assoziativen Aktivitäten ". ${ }^{37}$

Aufmerksamkeit, genauer „öffentliche Aufmerksamkeit" für die Probleme und Potentiale des öffentlichen Lebens gilt in Konzepten „politischer Kultur“ als entscheidende Voraussetzung einer kritischen öffentlichen Meinung. Öffentliche Aufmerksamkeit ist der sozialen Probleme und Potentiale der anderen und krisenhafter Entwicklungen des Gemeinwesens gewahr. Aktive Aufmerksamkeit ist dann bereit, die Kritik auch als Problem und Protest öffentlich zu machen und damit Kritik ins Konstruktive zu wenden, indem öffentlich Bereitschaft signalisiert wird, bei einer Veränderung der Verhältnisse mit-arbeitend und mit-verantwortlich aktiv zu werden.

Vertrauen bedeutet in einer, schwachen ${ }^{6}$ Variante die Überwindungen von Angst und Mißtrauen, womit erst Offenheit im sozialen wir im öffentlichen Leben möglich wird. Dies stärkt die ,starke Variante von Vertrauen im Sinne der Offenheit für aktive Kooperation mit anderen Beteiligten und Betroffenen.

Vernetzung setzt in ihren assoziativen Aktivitäten das ,soziale Kapital' aktiver „Aufmerksamkeit" und aktiven „Sozial-Vertrauens" voraus. Das gilt für die primäre Nähe der persönlichen und höchstpersönlichen informellen Beziehungen, es gilt aber auch für die über die Medien Geld und Macht hochformalisierten Beziehungen in wirtschaftlichen oder auch politischen Organisationen. Eine ganz andere Qualität aktiver Vernetzung entwickelt sich im ,zivilen Sektor" selbstorganisierten Engagements. Entscheidendes Potential der sozialen Aktivierung ist hier die kulturelle Sinnkonstruktion sozialen Engagements. Sinn-Fragen selbst-organisierten Engagements sind allerdings nicht mehr zu reduzieren auf die jeweils einfachen Eindeutigkeiten von partikularen ,Ehren und universellen ,Werten'. Vielmehr bedeutet selbstorganisiertes Engagement heute, daß auch die gemeinsame Sinnkonstruktion eines aktiven „Gemeinsinns" selbst erarbeitet und erörtert werden will. In solch kommunikativer Kultur verbindet sich die Solidarität selbstorganisierter Netze mit der Reflexivität von Lernprozessen einer offen verhandelten Sinnbildung des bindenden und bewegenden Engagements.

Genau in dieser Umstellung von den großen ,Werken' milieu-gemeinschaftlich gebundener Mitgliedschaft zum reflexiven Engagement neuer Netzwerke und Lernprozesse liegt aber auch ein Dilemma des Gemeinsinns: Mit den aktuell im Dritt-Sektor beob-

${ }^{35}$ Vgl. Putnam/Gross 2001, S. 20 f.

${ }^{36}$ Ebd., S. 22.

37 Offe/Fuchs 2001, S. 418. 
achtbaren Entwicklungen wird das „soziale Kapital“ der Gesellschaft nicht nur verlagert, sondern auch neu gemischt und geteilt. So erkennen wir nicht nur neue Entwicklungsperspektiven bürgerschaftlichen Engagements sondern auch neue Fronten kultureller Trennung und sozialer Spaltung:

Der „Schwund des Sozialkapitals" der großen Massenorganisationen und die Umstellung von alter Ehre auf neues Engagement, von Rationalität auf Reflexivität macht das Engagement gewiß anspruchsvoller. Waren die älteren Großverbände mit ihren flacheren Eintrittsschwellen offen für alle, so haben wir es heute zu tun mit einem weit steileren Engagement mit steigenden Ansprüchen an Motivation und Kompetenz. Gerade dies aber bleibt nicht ohne Probleme: Der Umstieg ins reflexive Engagement hat spürbar selektive Effekte: Gerade die bildungsfernen Schichten, die massenhaft aus ihren älteren Milieu-Gemeinschaften herausfallen, finden dann nicht mehr den Einstieg über die ,gehobenen" Schwellen eines situativ und reflexiv organisierten neuen Engagements. Viele sehen sich hier eher abgestoßen und ausgeschlossen. Die alten Vereinigungen und neuen Initiativen bürgerschaftlichen Engagements sollten sich diesem „Dilemma des Gemeinsinns ${ }^{\text {c38 }}$ stellen, daß bei offensichtlich ungleichen Partizipationschancen einladende Hilfen zur Förderung von Partizipationsbereitschaft und Partizipationsfähigkeit entwickelt werden müssen, um einer sich ,spaltenden Bürgergesellschaft ${ }^{39}$ gegenzusteuern.

Dies fordert Reflexion, also Offenheit für wechselseitige Lernprozesse: „Die eigentliche Herausforderung besteht darin, institutionelle Arrangements zu entwickeln, welche Lernfähigkeit [...] belohnen." 40

\section{Literaturverzeichnis}

Anheier, H. K. (1999), Rahmenbedingungen für die Arbeit sozialer Organisationen, Initiativen und Dienste in: Organisationen, Initiativen und Dienste im sozialen Bereich - ein Motor der Sozialpolitik in Europa, hg. v. BmFSFJ, S. 16-22.

Baader, F. v. (1835): Ueber das dermalige Missverhältniss der Vermögenslosen oder Proletairs zu den Vermögen besitzenden Klassen der Societät in Betreff ihres Auskommnes, sowohl in materieller, als intellektueller Hinsicht, aus dem Standpunkt des Rechts betrachtet, München, zit.n.: Gesellschaftslehre, hg. v. E. Pankoke, Frankfurt/M. 1991, S. 320-339, 1079-1089.

Bertelsmann Stiftung (Hg., 2000), Handbuch Bürgerstiftungen. Ziele, Gründung, Aufbau, Projekte, Gütersloh.

Brömme, N./Strasser, H. (2001), Gespaltene Bürgergesellschaft? Die ungleichen Folgen des Strukturwandels von Engagement und Partizipation, in: Aus Politik und Zeitgeschichte, Bd. 25-26, S. 6-14.

Brückers, R. (1999), Qualität der Arbeit sozialer Organisationen, Initiativen und Dienste: Perspektiven der Zusammenarbeit in Europa - Qualitätsstandards, in: Organisationen, Initiativen und Dienste im sozialen Bereich - ein Motor der Sozialpolitik in Europa, hg. v. Bundesministerium für Familie, Senioren, Frauen und Jugend, Bonn, S. 75-80.

Erlinghagen, M. (2001), Die sozialen Risiken „Neuer Ehrenamtlichkeit“. Die Zukunft des Ehrenamtes am Beispiel der „Bürgerarbeit", in: Aus Politik und Zeitgeschichte, Bd. 25-26, S. 33-38.

38 Vgl. Joas 2001.

39 Vgl. Brömme/Strasser 2001.

${ }^{40}$ Kaufmann 1992, S. 114. 
Feurt, S. L. (1999), Vorbilder, Erfahrungen und Modelle. Bürgerstiftungen in internationaler Perspektive, in: Community Foundations in Civil Society/Bürgerstiftungen in der Zivilgesellschaft, hg. v. Bertelsmann Foundation, Gütersloh, S. 137-155.

Heidegger, M. (1986), Sein und Zeit, Tübingen.

Herrmann-Pillath, C./Lies, J. J. (2001), Soziales Kapital - ein öffentliches Gut?, in: Sociologia Internationalis 39 (i.E.).

Jakob, G. (1993), Zwischen Dienst und Selbstbezug. Eine biographieanalytische Untersuchung ehrenamtlichen Engagements, Opladen.

Joas, H. (2001), Ungleichheit in der Bürgergesellschaft. Über einige Dilemmata des Gemeinsinns, in: Aus Politik und Zeitgeschichte, Bd. 25-26, S. 15-23.

Kaufmann, F.-X. (1992), Der Ruf nach Verantwortung. Risiko und Ethik in einer unüberschaubaren Welt, Freiburg.

Luhmann, N. (1996), Protest. Systemtheorie und soziale Bewegungen, hg. v. K.-U. Hellmann, Frankfurt/M.

Marx, K./Engels, F. (1983), Zur Judenfrage, in: MEW, Bd. 1, Berlin, S. 347-377.

Münch, R. (1999), Europäische Identitätsbildung. Zwischen globaler Dynamik, nationaler und regionaler Gegenbewegung, in: Identität und Moderne, hg. v. H. Wiliems u. A. Hahn, Frankfurt/M., S. $465-486$.

Offe, C./Fuchs, S. (2001), Schwund des Sozialkapitals? Der Fall Deutschland, in: Gesellschaft und Gemeinsinn. Sozialkapital im internationalen Vergleich, hg. v. R. D. Putnam, Gütersloh, S. $417-$ 514.

Otto, L. (1991), Die Demokratinnen [1849/50], in: Gesellschaftslehre, hg. v. E. Pankoke , Frankfurt/M., S. 530-553.

Pankoke, E. (1966), Sprache in ,sekundären Systemen`. Zur soziologischen Interpretation sprachkritischer Befunde, in: Soziale Welt 17, S. 253-273.

Pankoke, E. (1994), ,Enthusiasmus‘ und ,Dilettantismus‘. Gesellschaftlicher Wandel ehrenamtlichen Engagements, in: Ehre. Archaische Momente in der Moderne, hg. v. L. Vogt u. A. Zingerle, Frankfurt/M., S. 151-171.

Pankoke, E. (1998), Stiftung und Ehrenamt, in: Bertelsmann-Stiftung 2000, S. 637-670.

Pankoke, E. (2000), Freie Assoziation. Geschichtliche Prämissen und gesellschaftliche Perspektiven moderner Genossenschaft, in: Engagierte Bürgerschaft. Traditionen und Perspektiven, hg. v. A. Zimmer u. S. Nährlich, Münster, S. 189-211.

Pankoke, E. (2001), Wertedynamik und Sozialmanagement. Qualitätskontrollen, Motivationskulturen, Evaluationsprozesse im Dritten Sektor, in: Modernisierung - Prozess oder Entwicklungsstrategie, hg. v. H. Hill, Frankfurt/M., S. 199-204.

Pankoke, E./H. Nokielski/T. Beine (1975), Neue Formen gesellschafticher Selbststeuerung. Diskussion an Beispielen aus den Bereichen Bildung, Soziale Sicherung und Kommunale Selbstverwaltung, Göttingen.

Putnam, R. D. (Hg., 2001), Gesellschaft und Gemeinsinn. Sozialkapital im internationalen Vergleich, Gütersloh.

Putnam, R. D./Gross, K. A. (2001), Einleitung, in: Gesellschaft und Gemeinsinn. Sozialkapital im internationalen Vergleich, hg. v. R. D. Putnam, Gütersloh, S. 15-43.

Schmid, J. (1996), Wohlfahrtsverbände in modernen Wohlfahrtsstaaten. Soziale Dienste in historischvergleichender Perspektive, Frankfurt/M.

Seibel, W. (1992), Funktionaler Dilettantismus. Erfolgreich scheiternde Organisationen im ,Dritten Sektor' zwischen Markt und Staat, Wiesbaden.

Stein, L. v. (1921), Geschichte der sozialen Bewegung von 1789 bis auf unsere Tage [1850], 3 Bde., hg. v. G. Salomon, München.

Stein, L. v. (1869), Das System des Vereinswesens und des Vereinsrechts, Stuttgart. 
Strachwitz, R. Graf (Hg., 1998), Dritter Sektor - Dritte Kraft. Versuch einer Standortbestimmung, Stuttgart.

Strachwitz, R. Graf (2000), Gründung, Aufbau und Organisation von Bürgerstiftungen, in: Bertelsmann-Stiftung 2000, S. 111-134.

Taliani, E. (Hg., 1996), Marginalità sociale e nuova Progettualità. Riflessioni, metodologie, esperienze, Pisa.

Trube, A. (1997), Zur Theorie und Empirie des Zweiten Arbeitsmarktes. Exemplarische Erörterungen und praktische Versuche zur sozioökonomischen Bewertung lokaler Beschäftigungsförderung, Münster.

Wagner, A. (1879): Allgemeine und theoretische Volkswirthschaftslehre, Erster Theil: Grundlegung, Leipzig/Heidelberg.

Weber, M. (1964), Wirtschaft und Gesellschaft. Grundriß der verstehenden Soziologie, hg. v. J. Winkelmann, Köln/Berlin. 
